\title{
LAS PRÁCTICAS DE MATERNAJE DE LAS MADRES CUIDADORAS DE LA CULTURA QOM COMO CONTENIDOS PARA LA FORMACIÓN EN PERSPECTIVA INTERCULTURAL
}

\section{THE MOTHERING PRACTICES OF THE CAREGIVER MOTHERS OF THE QOM \\ CULTURE AS CONTENT FOR TEACHER TRAINING IN AN INTERCULTURAL PERSPECTIVE}

\author{
Sylvia Edith Sandoval ${ }^{1}$ - Amancia Silvestre ${ }^{2}$ - Annek Shawny Zamora Aray
}

Fecha de recepción: 18-04-2021

Fecha de aceptación y versión final: 09-10-2021

\section{Resumen}

El artículo pretende reflexionar sobre resultados de experiencias del proyecto de investigación "Las Prácticas de maternaje de las Madres Cuidadoras de la Cultura Qom" con contenidos sobre historia familiar y de la comunidad de la asignatura Ciencias Sociales en la Educación Inicial del Profesorado en Educación Inicial. Se trata de un estudio de caso en perspectiva intercultural, en contextos sociales con pueblos indígenas que indaga prácticas utilizadas por las MCCQ en la crianza de los niños y niñas qom de su comunidad a partir de las cuales construyen la noción de historia familiar y de la comunidad. Se elaboró un marco conceptual, que propone categorías que analizan las características que adquieren las dimensiones tiempo y espacio en la narración del pasado. En el contexto de diversidad, el caso testigo permitió contrastar el modelo generalizado de enseñar la historia familiar en la cultura criolla y resaltar distintivamente el vínculo de la narración del pasado con el entorno natural. Empero, estos contrastes, llaman a revisar modelos hegemónicos sobre narraciones del pasado y lugares de memoria comunitaria. La reflexión sobre ambos aspectos debe estar presente en la formación para promover el ejercicio de la ciudadanía inclusiva en el jardín de infantes.

Palabras clave: Madres Cuidadoras de la Cultura Qom - Interculturalidad - Memoria-territorio Historia familiar.

\begin{abstract}
The article aims to reflect on the results of the research project " Mothering Practices of Caregiver Mothers of the Qom Culture" (CMQC) and contrast them with the contents on family and community history delivered by the Chair of Social Sciences in Early Childhood Education of the Early Education Teacher's Degree program. It is a case study in social contexts with indigenous peoples that investigates practices used by Caregiver Mothers in the upbringing of Qom children in their community. The notions of family history and community stem from these practices.

To this end, a theoretical framework with an intercultural stance was developed. We also proposed categories to analyze the characteristics of time and space in the narration of the past. In a context of socio-cultural diversity, the case study allowed us to contrast the Qom model with the generalized model of teaching family history in Creole culture and highlight the link between the narration of the past and the natural environment. However, these comparisons call for a review of hegemonic models of past narratives and places of community
\end{abstract}

\footnotetext{
${ }^{1}$ Facultad de Humanidades, Universidad Nacional del Nordeste. Argentina. Correo electrónico: mausidos@gmail.com

${ }^{2}$ Madres Cuidadoras de la Cultura Qom. Correo electrónico: madrescuidadora@gmail.com

${ }^{3}$ Facultad de Humanidades, Universidad Nacional del Nordeste. Argentina. Correo electrónico: grighots@gmail.com 
RIIE (2021), Año 12 (16), 98- 114.

LAS PRÁCTICAS DE MATERNAJE DE LAS

DOI: http://dx.doi.org/10.30972/riie. 13165760 MADRES CUIDADORAS DE LA CULTURA QOM...

memory. Reflection on both aspects must be present in teacher education to promote the practice of inclusive citizenship in kindergarten.

Key words: Caregiver Mothers of the Qom Culture - Interculturality - Memory-territory - Family History. 


\section{Introducción: Problema de investigación, objetivos y metodología del estudio}

El contexto de estudio tuvo lugar durante tres etapas del proyecto de investigación que van del 2010 al 2018. Cabe aclarar, que el mismo tiene sus antecedentes en experiencias extensionistas iniciadas en el 2007 vinculadas con demandas puntuales de las Madres Cuidadoras de la Cultura Qom -en adelante MCCQ- relacionadas a la educación intercultural bilingüe con pueblos indígenas, particularmente con las prácticas de crianza. Para el artículo se seleccionó una de estas demandas provenientes de situaciones de enseñanza ocurridas en los jardines de su localidad, vinculada con la transmisión de la historia familiar y con las características que adquiere la narración del pasado en la cultura qom.

La historia familiar, forma parte de los contenidos mínimos de la asignatura Ciencias Sociales en la Educación Inicial fundamentados en el marco de la diversidad cultural. Con el propósito de incorporar estos saberes de la cultura qom a la formación realizamos los registros de campo correspondientes. Durante los encuentros, se observaron diferencias con la cultura hegemónica: en primer lugar, las MCCQ narran la historia familiar con una profundidad temporal que abarca varias generaciones, ampliando el relato con experiencias y acontecimientos que involucran a la comunidad. En contraste, la historia familiar que se enseña en los jardines de los criollos se circunscribe a la generación de los padres y abuelos, y las efemérides equivalen a la historia de la comunidad. En segundo lugar, la narración de las experiencias del pasado estaba fuertemente vinculada al entorno natural, en contraste con el modo de narrar el pasado en la cultura criolla. Teniendo presente estas expresiones y en un contexto intercultural con pueblos indígenas, la asignatura Ciencias Sociales en la Educación Inicial reconoció la necesidad de revisar y reflexionar sobre aspectos importantes para concebir y transmitir la historia familiar y de la comunidad, adecuándolos a las culturas, para evitar afectar la identidad de las niñas y los niños indígenas, en particular si entran en conflicto con las prácticas de crianza qom. Algunas preguntas orientaron la indagación:

¿Qué papel cumplen las prácticas de maternaje? ¿Qué atributos pueden encontrarse en el modo de narrar el pasado, y de modo particular, la historia familiar y de la comunidad? ¿Qué elementos aportan las prácticas de maternaje de las MCCQ para dialogar entre culturas, semejanzas y diferencias de las historias familiares y de la comunidad?

\section{Objetivos:}

Presentar los marcos conceptuales para el análisis en perspectiva de diálogo intercultural en contextos sociales con pueblos indígenas.

Construir categorías de trabajo, en traducción intercultural, que den cuenta de las características de crianza y las dimensiones temporo-espaciales presentes en la historia familiar y comunitaria.

Reflexionar sobre la importancia de revisar críticamente los contenidos de la formación, teniendo presente su impacto en la enseñanza en el jardín en contextos diversos. 


\section{Metodología}

En un contexto de diversidad cultural, en el que se pretende visibilizar el diálogo intercultural con pueblos indígenas, las prácticas de maternaje de las MCCQ son consideradas como estudio de caso. La singularidad de las pautas de crianza seleccionadas por ellas para cuidar y enseñar la cultura qom en la generalidad del discurso y de los dispositivos de enseñanza de la cultura hegemónica blanca, la convierten en un modelo para orientar la revisión de contenidos y prácticas en lo concerniente a educación intercultural con pueblos indígenas. El abordaje cualitativo es de corte participativo, asistido por el registro etnográfico, en un ir y venir de intercambios entre los integrantes del equipo docente y las MCCQ. La información obtenida corresponde a registros de trabajo de campo, informes de proyectos de investigación como de extensión realizados durante la última década. Participaron algunas integrantes de las MCCQ como del equipo docente. Se prestó cuidado a la experiencia de las MCCQ, sus conocimientos y el reconocimiento de la comunidad al trabajo que ellas realizan sobre la historia familiar y de la comunidad. En este proceso que anhela ser intercultural, el peso de un contexto cultural hegemónico-blanco se filtra de modo permanente en los intercambios. Los encuentros se dieron en un ambiente de cordialidad y respeto donde la escucha y la palabra son predominantes en la cultura qom, como los gestos y los silencios.

En la escritura perdemos, evidentemente una inocencia; no porque la palabra sea por sí misma fresca, natural, espontánea, verídica, expresión de una interioridad pura, por el contrario (sobre todo en público) es inminentemente teatral, pide prestados sus giros (en el sentido estilístico y lúdico del término) a todo un conjunto de códigos culturales y oratorios: la palabra es siempre táctica, perceptible para quien sabe escuchar, como otros saben leer (Barthes, 2005, p. 9).

En las modalidades de entrevistas y conversatorios se registraron historias de vida, relatos ancestrales, etnojuegos, dibujos. Se complementó con estudios especializados pertinentes con la temática que se presenta. Los datos fueron aportados por integrantes de MCCQ (Juana Silvestre, Aureliana González, Amancia Silvestre, Sonia García). Por su parte, la asignatura toma la situación presentada por las MCCQ para contrastar con la noción hegemónica de historia familiar y de la comunidad desde el paradigma de la diversidad cultural y el diálogo intercultural con el propósito de visibilizar las concepciones diferentes de otras culturas. Acorde a ello, se seleccionó el módulo 2 del programa de la asignatura de Ciencias Sociales en la Educación Inicial (Cultura como proceso dinámico y diverso: diversidad cultural, interculturalidad, el caso de las MCCQ). El aporte entre lo trabajado en investigación con los contenidos del programa permitió el intercambio y reflexión para actualizar y profundizar saberes para la enseñanza en estos contextos.

A continuación, los puntos 1 y 2 del presente artículo exponen los marcos conceptuales sobre interculturalidad y traducción intercultural que orientan y posicionan teórica, filosófica y metodológicamente la investigacción. En este sentido, se pondrá foco en los principios sobre interculturalidad, para continuar con las características de traducción intercultural. El punto 3 presenta las categorías de trabajo con las que se delimitan los atributos de crianza propios de las MCCQ y el modo de narrar el pasado -trabajo de memoria-historia- en traducción intercultural, con la que se interpreta la historia familiar y de la comunidad. A partir de estas categorías, el punto 4 , destaca la historia familiar y de la comunidad narrada por las MCCQ y el uso de 
símbolos fuertemente ligados al entorno vital. Estos dos últimos puntos entran en diálogo y contrastan con nociones hegemónicas en la cultura criolla. De este modo, se pretende reflexionar y enriquecer los contenidos de la asignatura Ciencias Sociales, correspondientes al módulo 2 "Cultura como proceso dinámico y diverso", principio a partir del cual se propone enseñar en contextos de diversidad.

\section{Interculturalidad}

La perspectiva intercultural que preside esta investigación propone indispensable la práctica de un diálogo intercultural con las fuentes y en el trabajo de campo. Este marco conceptual proveniente de la filosofía intercultural permitirá analizar y reflexionar sobre la importancia de las categorías seleccionadas en el trabajo de investigación para vincularlas con los contenidos de enseñanza de la asignatura. Esta perspectiva parte de la base de que:

Las culturas no constituyen entidades cerradas, esenciales y eternas 0 ahistóricas, sino que ellas pueden ser consideradas como haces de tradiciones más o menos estables que guardan relaciones de poder conflictivas entre sí, que se transmiten en procesos complejos, se consolidan en las instituciones culturales y poseen elementos de contextualidad y de historicidad, que las tornan susceptibles de cambios, vínculos con otros grupos culturales, sincretismo y hasta de desaparición. (Fornet-Betancourt, 2009, citado en Sandoval, 2018)

En la propuesta teórica de Fornet-Betancourt (2009) se destacan y resumen cuatro características de la noción de interculturalidad que recorren el análisis del presente estudio y fundamentan la importancia de vincular los contenidos de la formación desde esta perspectiva:1) La interculturalidad es una "alternativa éticopolítica" que activa la diversidad para evitar el empobrecimiento de la uniformización cultural de la humanidad de un mundo globalizado propio del occidentalismo cultural. Si bien es un mundo globalizado tecnológicamente, la interculturalidad propone un mundo donde convivan muchos otros mundos contextuales. 2) La interculturalidad implica una perspectiva en el "reordenamiento de las relaciones internacionales" constituida desde acuerdos entre Estados, comprometidos en acciones concretas a través de convenios y apoyos solidarios entre pueblos y sus autoridades culturales. 3) La interculturalidad es una "alternativa epistemológica, hermenéutica y metodológica" frente a un saber culturalmente dominante. Por esto su horizonte es una utopía - en el sentido marcadamente político- de la necesidad de recuperar las memorias cognitivas, que pluralizan la ciencia y que da un lugar real en el mundo a cada uno de los saberes que transmiten sus muchas culturas, generando acciones que complementan el reajuste político con el equilibrio epistemológico en el campo de la praxis cultural. 4) La interculturalidad es un "movimiento" que se corresponde con un proceso real de reivindicación de la justicia cultural, política, cognitiva, etc., por parte de las tradiciones culturales marginadas. (Betancourt, pp.22-23)

\section{Traducción intercultural}

Ricardo Salas-Astrain (2006) hace referencia al modelo de traducción, que permite acordar las reglas específicas necesarias para construir espacios comunes de intercomprensión, que den cuenta de dos códigos lingüísticos y culturales articulados 
en una 'fusión de horizontes' significativa que logre comprender los sentidos y significados en la forma más recíproca posible. Salas recomienda que los interlocutores vayan al diálogo preparados y que cada uno conozca algo de la cultura del otro.

En un plano histórico, esta idea es relevante para dar cuenta de los modos discursivos de los evangelizadores que asumieron las formas de contacto asimétrico, desde la Conquista hasta nuestros días. Este plano temporal expresa la multiplicidad de las configuraciones histórico sociales y culturales que permiten hacer un camino discursivo 'inter-culturas' que contribuya a la elucidación de la comprensión de sí y a la comprensión del otro, definiendo los procedimientos que se necesitan para asegurar la mutua comprensión desde los contextos discursivos donde brota la productividad del sentido. (Salas-Astrain, 2006, p.154)

Raimon Panikkar (2004) por su parte advierte la importancia que la interculturalidad adquiere en la construcción de una ciudadanía en el mundo actual, para lo que propondrá una traducción intercultural basada en "equivalentes homeomórficos" entre culturas.

Los equivalentes homeomórficos parten de la percepción, en cierta manera racional, en cuanto la equivalencia presupone una especie de denominador común que la razón puede captar. En este sentido, la percepción de la armonía desafía los cánones de la racionalidad sin ser irracional. La intuición de la armonía descubre que existe un "algo" entre las partes y el todo que nos permite "experienciar" que se pertenecen, que encajan la una con la otra, aunque no sean semejantes o equivalentes. (Panikkar, p. 37)

De esta manera propone la superación del pensar racional introduciendo un pensar simbólico basado en la experiencia o agencia con el medio, la preferencia de pensar analógicamente y trabajar desde el valor epistémico de la oralidad, contrastando con el saber ontologizado de la escritura (Sandoval, 2018, p. 12). Desde una construcción sociológica centrada en lo político, De Souza Santos (2009) aportará a la traducción intercultural su análisis de lo que llama "situaciones de isomorfismo" de intereses entre las culturas en diálogo intercultural. Las situaciones isomórficas propuestas tienen una similitud con la propuesta de Panikkar, pero remiten a la esfera política del ejercicio de la interculturalidad. Los actores culturales involucrados en determinadas circunstancias de disputa deben acordar sobre cualquier aspecto que sea de mutuo interés, teniendo en cuenta la semejanza de los mismos y los intereses que se encuentren afectados. Es necesario destacar como sostiene Fornet-Betancourt (2009), que la interculturalidad es una alternativa epistemológica para recuperar las memorias cognitivas, pluralizar la ciencia y lograr el reajuste político en el campo de la praxis cultural. En cuanto a la traducción-interpretación, Salas-Astrain (2006) enfatiza la necesidad de construir espacios comunes de intercomprensión que articulen una 'fusión de horizontes' significativa para comprender los sentidos y significados en la forma más recíproca posible. Esto se refuerza con Panikkar (2004) al sostener que existe un "algo" entre las partes y el todo que encajan la una con la otra, aunque no sean semejantes o equivalentes, y los intereses en juego que afectan el ejercicio de los derechos políticos planteado por De Souza (2009). 


\section{El caso de las MCCQ y su aporte para pensar con perspectiva de educación intercultural los contenidos de Ciencias Sociales en la Educación Inicial.}

\subsection{Categorías de trabajo: Prácticas de Maternaje y Memoria-Territorio vivido}

\subsubsection{Prácticas de Maternaje}

Para las MCCQ, es fundamental la crianza de los niños y niñas en la lengua materna -qom la' aqtac- como el acompañamiento de la comunidad en esta etapa. El papel de la madre es irremplazable tanto como el período extendido de lactancia, lo que genera una relación muy estrecha con el niño y la niña. Luego de profundizar, interpretar e intercambiar significados con las MCCQ surgió para el caso de estudio la categoría específica "prácticas de maternaje":

Las prácticas de maternaje corresponden a la madre y deben ser aprehendidas y transmitidas de modo individual y comunitario a las hijas e hijos desde que nacen hasta los 10 años aproximadamente. Expresan las interacciones de todos los integrantes de la comunidad, conformando la trama del tejido social con facultades, limitaciones y obligaciones. La lengua, el tiempo comunitario vivido, el mito, los relatos, los preceptos y las enseñanzas, los tabúes, la familia, el cuidado de los hijos, la alimentación, los juegos, los espacios y los lugares son entre otras, sus expresiones materiales. Los bienes culturales transmitidos a través de las prácticas de maternaje se sostienen en la memoria y el territorio vivido. (Sandoval, 2013, pp. 50-51)

Aquí el argumento de Panikkar (2004), "existe un "algo" entre las partes y el todo que encajan la una con la otra, aunque no sean semejantes o equivalentes" sugiere que muchos de esos atributos son compartidos en la crianza de otras organizaciones socioculturales con sus diferencias y valores morales. Si se tiene como premisa que realizar determinadas actividades culturales genera hábito y con su repetición se desarrollan destrezas y habilidades, en cada organización sociocultural, en un tiempo y espacio particular, las equivalencias y /o semejanzas están presentes en la distinción de lo diverso. Algo semejante ocurre en la cultura criolla, en los primeros años de vida niños y niñas aprenden valores y normas que regulan su comportamiento y otorgan significado al mundo. También desarrollan capacidades y destrezas vinculadas a la lengua materna -español- como jergas propias de cada subcultura. Este primer entorno de aprendizaje ocurre por lo general en el espacio doméstico con sus propias regulaciones -alimentación, horarios, juegos, interacción con los integrantes de la familia, etc.- y prepara para transitar el espacio amplio del vecindario, de la ciudad o del pueblo -entender las señales del semáforo, distinguir medios de transportes y funciones, comportarse en el jardín, etc.- La interacción en ambos espacios complejiza la visión del mundo del niño y de la niña. Este proceso corresponde al concepto de endoculturación, contenido que se desarrollan en el módulo 2 del programa de la asignatura. De este modo, en traducción intercultural, la categoría puede encontrar equivalencias y similitudes de crianza.

La siguiente categoría, intrínsecamente ligada a las prácticas de maternaje es Memoria -territorio vivido. Como en la construcción de la categoría anterior, la perspectiva intercultural -y la pertinencia epistémica- de la que provienen estos supuestos surgen del pensamiento y trabajo conjunto con las MCCQ. Esto es, de los significados que atribuyen a las nociones sobre el pasado, la memoria y la historia 
vividos en el qaralamaxat qarma' y evocados por la transmisión oral, práctica autorizada a los sabios y sabias de la comunidad, como lo son las MCCQ. Las narraciones orales entrelazan relatos de tiempos míticos, como acontecimientos ancestrales que se extienden a la conquista, al poblamiento del Chaco y al tiempo de sus mayores. Empero, la historia del presente atraviesa los relatos de las historias de vida de las MCCQ, hunden sus recuerdos en sus memorias de crianza, a injusticias y expropiaciones padecidas en sus familias, a carencias básicas para subsistir y a las luchas por los derechos conculcados.

\subsubsection{Memoria-Territorio vivido}

Esta categoría pretende expresar la relación de la memoria con las experiencias vividas en el territorio, en el tiempo y en el espacio. El carácter de dimensión tiene su correlato con la magnitud real del Qaralamaxat Qarma' Etaxat Nuestros Lugares y entornos de agua- que constituyen el territorio de las MCCQ y sus familias extensas. En la actualidad abarca aproximadamente cien kilómetros.

Este Territorio nombrado y significado por nuestros mayores a través de generaciones antes de la llegada del blanco y del criollo a esta región, habla de una relación muy particular del Pueblo Qom con el espacio físico y sus recursos de los que ellos disfrutaron con libertad, sin dañarlo y con profundo respeto a la Madre Tierra. Este fue y es Nuestro Lugar en el Gran Chaco Argentino, porque nuestros antepasados poblaron esta región hace más de 7 mil años aproximadamente. Ellos recorrieron libremente este territorio, se apropiaron del espacio en el que habitaban y fueron dando nombres a los diversos lugares y a los hechos que allí sucedían. Los nombres que hemos recogido de nuestros ancianos y ancianas que viven todavía entre nosotras, son producto del relato vivo de nuestro pueblo y de sus gentes. (MCCQ, 2009, p. 8)

Tal vinculación expresa el sentido amplio biogeográfico y por tanto no reducido a la delimitación política. Por ejemplo, en los relatos de vida de las MCCQ como en los relatos provenientes de la literatura oral indígena (Silva, 2005), las experiencias expresan el vínculo de los qom con los entornos de vida, en el monte, en los ríos y lagunas. Las huellas de la memoria están fuertemente ligadas al territorio del Chaco, los qom cuentan su pasado mitológico, ancestral y reciente, a través del registro oral o escrito. De modo análogo, Annek Shawny Zamora Aray, perteneciente a la etnia wichi y egresada adscripta a la asignatura, refuerza esta posición:

A través de los años, los pueblos indígenas hemos vivido en armonía con la naturaleza, esta relación nos ha permitido subsistir a lo largo del tiempo, porque además de crear un estilo vida es también la base de nuestra cosmovisión. El equilibrio de la vida comunitaria se basa en este lazo de respeto por el otro, sean animales, plantas o seres humanos. Todos los seres formamos parte de una gran comunidad, en este círculo de vida convivimos. La espiritualidad, surge de esta interacción vital, está unida a todo. Las acciones individuales sean benéficas o dañinas afectan a toda la comunidad de vida. Esta concepción recibí de mi padre y de mi madre, de mis abuelas y de mis abuelos, de mis tíos y de mis tías. Llegaron como experiencias vividas por mis mayores y como memorias ancestrales. 
Desde la primera infancia, nuestra educación materna y familiar ha trabajado desde esos principios. Agradecemos a cada ser vivo por lo que nos brinda ya que en la naturaleza encontramos todo lo necesario para vivir, desde alimento hasta medicina y abrigo, en el monte está nuestro hogar, por lo menos así era en el principio, luego de los diferentes procesos de colonización y destrucción de nuestras comunidades la realidad es diferente. (Khey -Costumbres-, 2019, inédito)

En ese territorio -Qaralamaxat Qarma'- los qom y otros pueblos indígenas aprendieron a vivir, a conocer su entorno y simbolizarlo. En los fragmentos que a continuación se destacan pueden apreciarse experiencias ocurridas en este hábitat. En una de ellas, las MCCQ recordaban algunas formas de defenderse de animales que les transmitieron sus madres:

Nuestras madres y mayores iban al monte y se encontraban con animales de antes como el oso hormiguero, el oso tiene garras y ataca a matar, entonces con un palo largo le pegan en el hocico, el único lugar que tiene para pegarle y es muy sensible. (Juana Silvestre y Amancia Silvestre, comunicación personal, Pampa del Indio, agosto de 2007)

La recolección de frutos era una actividad comunitaria, aprovechaban todo el año, en primavera en el monte y en el río se conseguían alimentos en abundancia, la siguiente es una traducción del qom:

Margarita, mi madre recuerda que anteriormente se iban allá, a la costa del rio Bermejo los días que maduran la fruta del chaguar. Entonces dice que las mujeres comprobaron las dos clases de fruta de chaguar que existen. Dicen que, en esta parte, la fruta es agridulce, pero ellas quisieron ir hacia el otro lado del río y encontraron otra fruta más buena, entonces se pusieron de acuerdo unas cuantas mujeres y cortan ramas de sauce, atan con fibra de Palo Borracho y arman como una camilla que puedan resistir el agua. Lo atan muy bien, cuando está todo hecho cargaban sus yicas y las mujeres nadan al otro lado, van empujando al otro lado del rio, eso le sirve para que al llegar al otro lado pongan la fruta allí, es como una balsa. Encontraron de ese lado un fruto más dulce para comer, el de acá es más agrio y no tan bueno para comer. (Margarita Rojas y Juana Silvestre, comunicación personal, Pampa del Indio, agosto 2007)

Estos fragmentos seleccionados indican una fuerte relación con el entorno natural proveniente de las experiencias adquiridas en la crianza. Corresponden a las historias de vida de las MCCQ, a las memorias de la niñez y a la crianza que aún transmiten los mayores a los niños y niñas de su comunidad.

\subsection{El análisis desde la perspectiva intercultural}

De igual manera que el hábito del saber hacer cotidiano constituye las prácticas de maternaje, las huellas mnemónicas de estas experiencias forman la base de la Memoria-territorio vivido. Para analizar el pasado, se parte de este umbral, que Ricoeur (2008) llama memoria cognitiva y a partir de esta se avanza hacia la memoria que busca en el pasado para ser transmitida. Se reconocen dos clasificaciones de memoria trabajadas, una de ellas es constitutiva de las prácticas de maternaje o de 
crianza en cualquier cultura (...) "se refiere a la memoria natural, adiestrada con las actividades necesarias para la cotidianeidad de la existencia biológica-social y otra a la memoria artificial, sostenida por la intencionalidad de recordar, de llamar al pasado, de ir en su búsqueda" (p. 81). La relación entre los dos tipos de memoria es importante para comprender la importancia que las prácticas de maternaje de las MCCQ tienen en la constitución de la historia familiar y de la comunidad.

La memoria natural surge y se sostiene en el hábito, a partir de un modo de actuar "no marcado", en tanto resulta marcado para la memoria específicamente tal. Si bien la memoria, se vincula con el pasado, el hábito como experiencia recurrente, también atraviesa el tiempo. El hábito se encuentra en una menor profundidad temporal dado que responde a experiencias que, una vez incorporadas, son actuadas de modo regular. (Sandoval, 2018, pp. 45-47)

En este caso, la memoria-hábito de Ricoeur (2008) es la memoria que se adiestra para actividades vinculadas a la subsistencia diaria, ir a pescar al tala -río para los qom-, armar una balsa, nadar y recoger frutos del monte, observar las huellas de animales en los senderos, conocer donde asestar golpes a determinados animales, colectar plantas medicinales o alimenticias, entre otras actividades que las MCCQ realizaron a diario junto a la comunidad y fueron parte de su propia crianza. Estas destrezas las desarrollaron desde edades muy tempranas, a su vez sus hijas las repiten con sus hijas e hijos. Aprendieron y aprenden a reconocer animales peligrosos por el tipo de huella, cómo defenderse, qué plantas recoger para fortalecer la lactancia, cómo limpiar de espinas los frutos de la tuna, qué hierba machacar para dormir a los peces y recogerlos en las redes, entre otros saberes que enseñan los modos de subsistencia en un territorio específico, el Qaralamaxat Qarma' Etaxat nuestros lugares y entornos de agua. Para profundizar en la historia familiar y de la comunidad, la memoria-hábito se complejiza en un segundo tipo de memoria (Ricoeur, 2008) Ilamada memoria artificial o memoria que imagina puesto que quienes narran memorias, justamente ejercen su función mediante la invocación intencional de los recuerdos.

Cuando queremos contar algo de nuestros ancianos, nosotras solemos llamar al pasado, porque allí están bien guardados los recuerdos de nuestras costumbres y de lo que ellos supieron hacer, escuchamos a nuestros libros vivientes que son los ancianos y ancianas, si ellos no están, entonces podemos narrar nosotras. (Juana Silvestre, 2008, citado en Sandoval, 2018, p. 85)

La categoría Memoria-territorio está intrínsecamente ligada a la categoría Prácticas de Maternaje. Hay recuerdo del pasado si hubo hábito o experiencia constituida. Desde esta condición se avanza al campo de la historia y de la enseñanza del pasado de la comunidad. Es impensable para los qom y las MCCQ representar la identidad cultural fuera de la relación entre territorio - lengua, creencias y valores a lo largo del tiempo. En traducción intercultural y en clave de educación intercultural: ¿podemos pensarnos y asumir una identidad cultural colectiva fuera de ese argumento? Los estudios sobre la memoria colectiva de Halbwachs (2004) se basan precisamente en los cuadros sociales de la memoria situados en un espacio y un tiempo, equivalentes a los atributos de memoria-territorio vivido. Si la diversidad cultural es un principio para enseñar, ¿se visibilizan sus expresiones? Si, como sostiene Salas-Astrain (2006), tenemos presente que "el modelo de traducción permite 
construir espacios comunes de intercomprensión", al habilitar instrumentos para el análisis en traducción entre culturas, las singularidades visibilizarán, enriquecerán y complejizarán las dimensiones del objeto de estudio de la enseñanza. Las nociones de equivalentes homeomórficos de Panikkar (2004) como la de isomorfismo de De Souza Santos (2009) que orientan la traducción intercultural son importantes para comprender lo que se pone en juego en la educación intercultural y en la enseñanza de la historia. El tema de la crianza, socialización, prácticas de maternaje u otra noción equivalente puede analizarse desde tales marcos conceptuales y tienen efecto real sobre la vida social. En esta dimensión se producen tensiones y conflictos de intereses entre las personas, las comunidades, los pueblos. La reparación y restitución de derechos conculcados a los Pueblos Indígenas se expresa en la Ley de Educación Provincial $\mathrm{N}^{\circ} 6691 / 10$, con los correspondientes diseños curriculares qom, wichi 0 moqoit. En términos De Souza Santos (2009) estos hechos nos hablan de "situaciones de isomorfismo". En este sentido, la ley reconoce lo que Fornet-Betancourt (2009) señala como valor epistémico de saberes de otras culturas. Se revaloriza para el Nivel Inicial y Primario la centralidad del juego, la socialización y la alfabetización cultural, aspectos fundantes de la identidad de las niñas y niños indígenas, como otros colectivos culturales.

\section{Historia familiar y de la comunidad}

Si se trata del pasado, las prácticas de maternaje tienen un papel insustituible en vincular las experiencias vitales con la memoria que recuerda. Para transmitir la historia familiar se utiliza la figura del Árbol Qom, símbolo compartido por muchas culturas lo que facilita el dialogo y por tanto la intercomprensión.

\section{Figura 1}

\section{Árbol de Familias Qom}

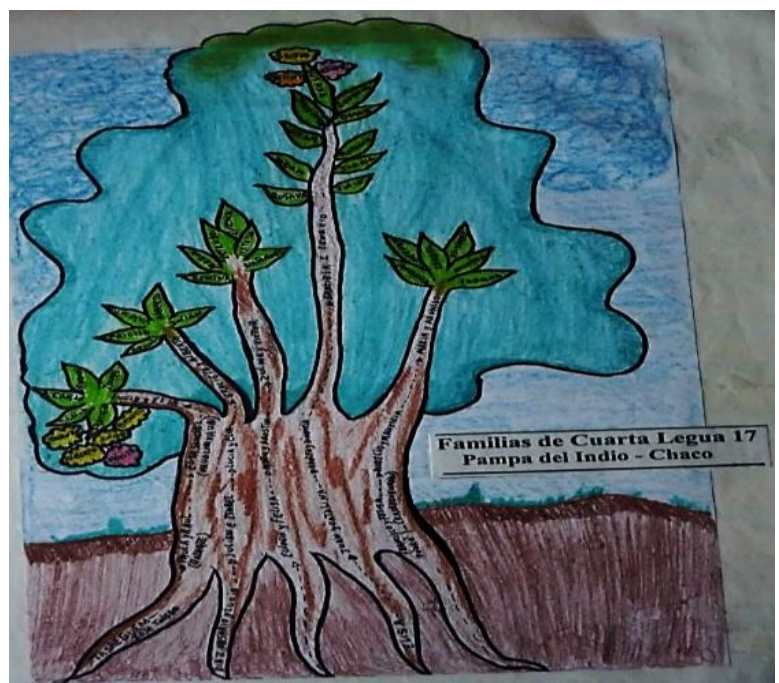

Fuente: Dibujo realizado por Las Madres Cuidadoras de la Cultura Qom (2007), inédito. 
Durante un trabajo de campo realizado en el 2009 en el Salón de las MCCQ en Pampa del Indio, se preguntó cómo estaban integradas las familias. Respondieron que sus familias a diferencia de las nuestras son muy grandes. Aureliana Gonzáles y Amancia Silvestre nos mostraron uno de los árboles de las familias de Cuarta Legua 17 distante a unos ocho kilómetros del Salón. Explicaron que los ancestros representan las raíces profundas fuertemente adheridas al Qaralamaxat Qarma' Etaxat -territorio-, en cada raíz hay uniones que provienen de historias entre familias de otros territorios. El tronco, corresponde a las uniones entre madre y padre, abuelas y abuelos y antepasados de más de cinco generaciones atrás. Las ramas representan las uniones matrimoniales de las MCCQ. Las hojas son sus hijas e hijos. Las flores sus nietas y nietos. Ante la insistencia que describieran quiénes integraban la familia de cada una, siguiendo el patrón cultural hegemónico, respondieron que entendían lo solicitado pero que para ellas la familia es la comunidad y así quieren que los criollos lo entiendan. A partir de esta convicción, las MCCQ enseñan a sus hijas e hijos que las familias son la comunidad, que las necesidades de la familia son de la comunidad, que los logros de las familias son también de la comunidad. En este nivel se ejecuta la "memoria del nosotros" (Ricoeur, 2008), aquí las prácticas de maternaje de las MCCQ comienzan su función historiadora, porque enseñan a través de las prácticas de maternaje la historia de su comunidad.

En traducción intercultural el árbol de las familias qom es uno de los tantos símbolos qom que da cuenta del ejercicio de la memoria activa, su uso pedagógico sirve para ilustrar la reconstrucción de la propia historia contada y vivida por los gom. El árbol es un símbolo para escriturar la historia de la comunidad. La figura del Árbol de las familias Qom guía la representación del mundo hacia la ascensión del saber. El árbol condensa en un lugar y en un tiempo el espacio sagrado (Elíade, 1998) de la genealogía familiar, al que concurren otros árboles familiares con sus genealogías configurando la narrativa de la historia de la comunidad.

Las MCCQ Ilaman "Árbol Qom" a las representaciones de las uniones familiares y su descendencia. La comunidad de los Qom Pi -gente qomtiene sus orígenes en lazos consanguíneos. La ilustración del árbol qom representa a las familias que componen la CQPI. A través de la historia oral se recuerda a los antepasados más cercanos que sufrieron la conquista del Chaco entre 1884 y 1885 . El árbol representa el tiempo y el territorio habitado por esta comunidad. Cuarta Legua lotes 17 y 14, son algunos de los lugares donde se asentaron definitivamente estas y otras familias qom de Pampa del Indio o -Piguiñiclae- en qom. El árbol cuenta de la voz de las MCCQ las narraciones del pasado dando testimonio de un territorio poblado, contradiciendo de esta manera la idea oficial de fines de siglo XIX hasta entrado el siglo XX de considerar al Chaco como un "desierto verde". (Sandoval, 2018, p. 155)

Lo que caracteriza el trabajo de memoria que realizan las MCCQ sería en términos de Ricoeur el "atributo de memoria ejercida" $(2008$, p.96). Este tipo de memoria se presenta como un primer nivel de reconstrucción de la memoria, de índole individual, que junto a otras memorias individuales, se lanzan a conformar las memorias familiares y luego la memoria comunitaria ingresando así a la historia de la comunidad.

Uno no se acuerda sólo de sí, que ve, que siente, que aprende, sino también de las situaciones mundanas, en la que se vio, se sintió, se 
aprendió. Estas situaciones implican el cuerpo propio y el cuerpo de los otros, el espacio vivido, en fin, el horizonte del mundo y de los mundos, bajo el cual algo aconteció. (Ricoeur, 2008, p. 57)

En la recuperación de la memoria como en la reconstrucción de la historia, juega un papel importante el arte de narrar el pasado de la comunidad, mantenida como práctica de los adultos y de los ancianos. La vigencia de la lengua madre ha conservado el acervo de la cosmovisión qom, pese a las transformaciones ocurridas en condiciones históricas de contacto. Para Ricoeur (2008a), el vigor de la lengua se expresa en la oralidad, por esta característica el testimonio oral adquiere relevancia epistémica. En lo que refiere a la memoria ligada al ámbito doméstico e intrafamiliar, las narraciones sobre juegos vinculados a las destrezas de crianza, desarrolladas en un primer nivel de configuración del hábito- memoria, son puestas en valor por las MCCQ a través de las prácticas de maternaje. De modo análogo, en la cultura hegemónica, la función socializadora de trabajar la historia familiar y de la comunidad en el jardín de infantes comparte el propósito de configurar la identidad colectiva, la pertenencia compartida del "nosotros", la transmisión de los valores, creencias y costumbres. En el terreno de lo político y en clave intercultural, las representaciones simbólicas del Árbol de las familias Qom como de Qaralamaxat Qarma' Etaxat pueden analizarse en tanto "lugares de memoria" (Nora, 1998) conformados por una realidad histórica y otra simbólica y como "memoria activa" en la medida que es activada por el sujeto, orientada en acciones que den sentido al pasado ante los problemas del presente (Jelin, 2002, p.23). Se trata de constituir un conjunto simbólico y advertir la intención que da cuenta del trabajo de memoria en su labor de evocación actualizada.

La memoria del nosotros -reflexividad/mundaneidad (Ricoeur, 2008)- que las MCCQ transmiten en su tarea pedagógica desde las prácticas de maternaje se presenta en el orden del espacio público, en el ámbito de la vida política, en las escuelas, en la universidad, en los centros culturales. En estos espacios se ponen de relieve las tensiones de los procesos de lucha por el reconocimiento de derechos y de legitimación de prácticas interculturales. También en estos ámbitos se disputan lo que De Souza Santos (2009) presenta como "situaciones de preocupaciones isomórficas" (p. 517) vinculadas con la tensión de intereses comunes entre las culturas, adscribiendo al criterio de Norá (1998), "al poner en el mismo plano el hecho y su conmemoración" (p. 22). El trabajo de reconstrucción de la memoria de la Comunidad Qom de Pampa del Indio -en adelante CQPI- ha convertido las figuras del Árbol de las familias Qom y del Qaralamaxat Qarma' - Etaxat en objetos de investigación histórica. De ahora en más las MCCQ y los idóneos de la CQPI decidirían el uso moralizante que orientará el análisis del pasado y, en este plano, su función pedagógica será decisiva. Se trata de un proceso que Jelin (2002) denomina "memoria activa" (p. 125).

El Árbol de las familias Qom se transmite como historia de la comunidad en las prácticas de maternaje. Este trabajo de memoria activa condujo a instalar en el terreno de lo político el reclamo por la reparación histórica y la restitución de derechos a la Comunidad Qom de Pampa del Indio. En esta dirección se condensan y expresan las demandas sociales que llevan al ámbito del espacio público las versiones del pasado vinculadas a las tensiones e intereses del presente y se expone el interés colectivo de los qom por transmitir el sentido reflexivo del pasado no sólo hacia adentro de su propia cultura, sino en sus relaciones con la cultura hegemónica. En tanto construcción que expresa la existencia de culturas distintas, el trabajo de recuperación de la memoria colectiva de la sociedad chaqueña trae aparejados conflictos de intereses, ya que las memorias indígenas disputan con las memorias de 
la cultura hegemónica las interpretaciones como la veracidad de los hechos del pasado.

Este mismo ejercicio de la memoria habilita lo que Ricoeur (2008) pondera como efectuación feliz cuyo propósito moral se dirige al empoderamiento del sujeto histórico, condición emancipatoria para vivir una interculturalidad política. Los contenidos de la asignatura permiten contrastar con los aportes del estudio, dado que la cultura hegemónica enseña la historia enfatizando las efemérides o conmemoraciones escolares entendidas como hechos y acontecimientos del pasado considerados importantes para la preservación del "nosotros" cultural. Es necesario recurrir a los diseños curriculares de los pueblos indígenas para incluir sus propias conmemoraciones, respetando su visión y construcción de la historia y en ella su concepción del "nosotros los pueblos indígenas". Es importante dar espacio a la historia a contrapelo de la historia legada, la historia reciente de los últimos treinta años se está abriendo camino de uno y de otro lado, es necesario escuchar las voces y ejercer memoria activa para interpelar el pensamiento único.

A través de las historias familiares y de las comunidades de ambas culturas se pueden reconocer y contrastar características vinculadas a integrantes y roles en las distintas familias. En la comunidad qom, aunque sus integrantes vivan lejos, en otra ciudad o provincia, la concepción de familia es la misma, incluso si hay segundas uniones entre el matrimonio. En el salón de las MCCQ hablamos sobre las características de familias blancas y de los qom:

Nuestras familias son grandes, además de nuestros hijos y marido están mis hermanos, mis sobrinas, mis primas y primos, mi madre y mis tíos. Si enviuda el hombre o la mujer y vive con otro, los hijos también son hermanos, como si fueran de la misma sangre. No decimos madrastra o padrastro, al menos antes era así. Pasa que en un mismo lote estamos los hermanos, ahora no es tan así. Algunos suelen irse a los barrios. (Aureliana Gonzáles, comunicación personal, Pampa del Indio, abril, 2008)

La forma de organización de la familia en la cultura qom y en el contexto de las MCCQ y en general en los pueblos indígenas se la concibe como familia extensa, constituida en principio por la madre, el padre, hermanos y hermanas, tíos, tías, primas, primos, abuelas, abuelos, que generalmente viven cerca. Es tan arraigada la memoria-territorio vivido que el sentido de familia extensa es enseñado a niños y niñas qom.

El jardín donde trabajo queda a 30 kilómetros del pueblo, es ahí donde los niños mantienen todavía su lengua materna, pero se cambió ahora en la actualidad. Yo soy la seño, la que está frente a la sala. Y como ellos saben que también soy de esa comunidad, que hablo el mismo idioma, entonces ellos por más que no te conozcan, por más que no sean tus parientes, me dicen tía, no me dicen seño. Tengo mi pareja pedagógica que siempre les dice, no es tía es su seño, pero para ellos y nuestra comunidad es natural porque es el respeto, más allá de que no sean tus parientes, saben que es parte de su comunidad que hablan la misma lengua, entonces te dan esa confianza, de tía. Yo tengo unos cuantos chicos que ya ingresaron en primaria y hasta hoy en día me dicen tía, por más que el docente (en qom) los corrige, claro y ellos saben que es su maestro, pero igual. En el caso de ustedes a las doqshe -blancas- le dicen señora o seño. Pero en el caso 
de nosotras es difícil, te dicen simplemente así tío, tía. (Sonia García, comunicación personal, Pampa del Indio, junio de 2017)

Cuando comenzamos en el 2003, nos invitaron que fuéramos las madres a trabajar con los chiquitos tobas en los jardines, porque son muy tímidos con la señorita porque no le entienden, porque la señorita blanca habla su idioma y los chiquitos tobas con otro y por ahí hay un desencuentro de palabras que no se entienden. Eso nos decían las señoritas que no tenían sus Auxiliares qom. Cuando llegábamos a las escuelas era toda una alegría para los chiquitos porque escuchaban el idioma que ellos hablaban en su casa. Al escucharnos a nosotros se ponían contentos, cosa que la señorita se reía porque no son así. Cuando llegábamos nosotros se ponían a cantar, a jugar, a contar cosas de la casa en nuestro idioma y nosotras le traducíamos a la señorita y eso le gustó mucho a ella. Algunos nos decían tías. (Amancia Silvestre, comunicación personal, Pampa del Indio, noviembre 2010)

En la cultura blanca la familia "tipo" está integrada por los progenitores y los hijos e hijas, no siempre incluye abuelos y abuelas, tíos, tías, primos, primas, por lo general no conviven permanentemente en el espacio familiar y no aparecen o son raros en los dibujos infantiles o en las narrativas sobre integrantes de la familia. Aparecen integrantes de familias ensambladas, pero también comienzan a visibilizarse las diversidades vinculadas con las identidades de géneros. En la zona rural del caso testigo la familia extensa se conserva, situación que permite contrastar para este estudio, las expresiones de la diversidad cultural. Las diferentes formas de organización y composición familiar son importantes para la formación en los niveles y modalidades de la enseñanza. En tal sentido y en traducción intercultural, las categorías presentadas aportan elementos para considerar la importancia de la familia extensa. Puede estar ausente la consanguinidad directa, pero los vínculos dados por la crianza en lengua materna y en su territorio ancestral al que llaman -Qaralamaxat qarma'- nuestros lugares, reveló que las familias constituyen una unidad más compleja para las MCCQ y los pueblos indígenas, las familias constituyen la comunidad.

\section{Reflexión final}

Partiendo del supuesto que la cultura es un proceso "vivo", dinámico y diverso, la perspectiva intercultural se convierte en el espacio apropiado para contrastar y poner en conflicto nociones, categorías, conceptos, valores, prácticas y creencias que forman parte de las identidades de las personas que aprenden en los distintos niveles educativos. Presentar las semejanzas, los contrastes, las diferencias en los ejemplos de los contenidos seleccionados nos permite contribuir a enriquecer el discurso educativo construido en una sociedad pluriétnica y pluricultural. En esta presentación podemos reconocer aspectos interconectados que tendrán efecto real en el ejercicio de la ciudadanía del presente. Estos refieren a la reparación histórica y a la restitución de derechos conculcados logrados mediante un proceso de diálogo entre sociedades culturalmente distintas. Las situaciones de isomorfismo deben garantizar en un litigio, las condiciones de igualdad entre las partes. En el caso de estudio se trata de reparar los daños causados por el etnocidio ejecutado por el estado y la destrucción simbólica ejercida por el discurso de la cultura hegemónica. Da cuenta de este proceso histórico de reparación y restitución, el acto pedagógico de las MCCQ 
cuando enseñan su propia historia a partir del trabajo de memoria, desde sus propias fuentes, predominantemente con la memoria oral pero también recurriendo a la memoria escrita y escribiendo desde sus propias interpretaciones. El acto educativo que enuncie la diversidad cultural deberá incorporar estas memorias diferentes y disidentes si se presume democrático e inclusivo.

La diferencia y la disidencia no restringen su aporte al campo de la ciudadanía, también contribuyen con la episteme del discurso educativo, en un ir y venir de fundamentos legitimados entre la ciencia y la política. Los atributos generales que puedan tener los conceptos de relatos, juegos, historias de las familias, instituciones, alimentación, crianza, socialización, presentes en el programa de una asignatura adquieren sentido e interés si se indagan otras formas posibles que tienen las sociedades humanas de expresarse culturalmente. Las categorías de trabajo presentadas intentan expresar que los saberes recuperados por las MCCQ propios de los pueblos indígenas y de otras culturas en general, provienen de la crianza, en la incorporación del saber hacer cotidiano, adquirido por las destrezas del hábito y con el trabajo de memoria. La riqueza está puesta en la perspectiva intercultural que da cuenta de lo otro culturalmente distinto. La mirada intercultural opone fondo y contraste en la dinámica de la diversidad. Si consideramos como único discurso del pasado aquel que habla de las memorias hegemónicas, fondo y contraste no existen. El peligroso camino del pensamiento único reduce el conocimiento del mundo.

El caso de las MCCQ llevado a la formación en el campo de la educación intercultural en contextos sociales con pueblos indígenas interpela el espacio académico, instala las problemáticas reales de lo que se enuncia como texto inerte en un documento curricular. La cultura, la historia familiar y de la comunidad, la diversidad, el cambio, la permanencia, como contenidos y objetos de estudio se tornan reales, dentro y fuera del aula. Los aportes de investigación y docencia situados en el campo epistemológico dialogan también con los saberes culturales de las y los estudiantes. En este sentido, es necesario contrastar la noción de familia y de historia que traen, que definen las disciplinas y que conciben las MCCQ. Las respuestas se convierten en temas de discusión y de análisis. El intercambio apela a distintas fuentes, como dibujos infantiles, las propias biografías y modos de formular preguntas sobre los modelos hegemónicos de familias, reconocer las grietas que aparecen en los modelos de familia, visibilizarlos a través del estudio. Preguntar qué es la comunidad, qué vínculos se establecen entre las familias y la comunidad, son interrogantes aparentemente simples que nos conducen a aventurarnos a indagar para enseñar. Los procesos de cambio cultural ocupan un tratamiento particular para comprender la igualdad de derechos en democracia. En este sentido, es importante el reconocimiento de las singularidades, como la distinción de las identidades y diferencias de los grupos sociales que comprenden el universo socio-político-cultural de crianza. El caso testigo de las Madres Cuidadoras de la Cultura Qom permite articular nociones estrechamente relacionadas e interdependientes como territorio, crianza y familia en las que el tiempo y el espacio son trabajadas como coordenadas esenciales y complejas de la vida social, particularmente el tiempo personal, familiar y social como el de la memoria colectiva. En traducción intercultural estos saberes visibilizan la importancia que tienen para ambas culturas incorporarlos como contenidos de la formación y de la enseñanza en la diversidad cultural como otras diversidades, presentes en los jardines de infantes. 


\section{Referencias bibliográficas}

Barthes, R. (2005). El grano de la voz. Entrevistas 1962-1980. Argentina: Siglo XXI editores.

Cámara de Diputados de la Provincia del Chaco (2010). Ley de Educación 6691/16. Ley de Educación Provincial. Capítulo XIII, Educación Bilingüe Intercultural. Artículos 87 y 88 . Resistencia, Chaco: Cámara de Diputados de la Provincia del Chaco. Diseño Curricular para el Nivel Inicial y Primario aprobado por Resolución № 10469 /12: Ministerio de Educación, Cultura, Ciencia y Tecnología de la provincia del Chaco. Recuperado de https://sinescuela.org/_media/argentina:argentina_ley_pcial_chaco.pdf.

De Souza, Santos, B. (2009). Sociología jurídica crítica: para un nuevo sentido común en el derecho, Madrid, España: Trotta.

Eliade, M. (1998). Lo sagrado y lo profano. Barcelona, España: Paidós.

Fornet-Betancourt, R. (2009). Tareas y propuestas de la Filosofía Intercultural. Aachen, Alemania: Wissenschaftsverlag Mainz.

Halbwachs, M. (2004). La memoria colectiva Memoria colectiva y memoria individual. Trad. Inés Sancho-Arroyo. España: Prensas Universitarias de Zaragoza.

Jelin, E., (2002). Los Trabajos de memoria. Madrid, España: Siglo XXI.

Madres Cuidadoras de la Cultura Qom, (2009). Relatos QOM (Tobas), 2ª edición, Córdoba, Argentina: Copiar.

Madres Cuidadoras de la Cultura Qom - Nate'elpi Nsoquiaxanaxanapi, (2010). Qaralamaxat Qarma' (Nuestros Lugares), Córdoba, Argentina: Copiar.

Nora, P. (1998). Memoria e Historia, Madrid, España: Marcial Pons.

Panikkar, R. (2004). Tres grandes interpretaciones de la interculturalidad. Conferencia inaugural: En: Fornet-Betancourt, R. Interculturality, Gender and Educa-tion; Interkulturalität, (pp. 27-43). Frankfurt, Alemania.

Ricoeur, P., (2008). La Memoria, la Historia, el Olvido. Buenos Aires, Argentina: Fondo de Cultura Económica.

Salas-Astrain, R. (2006). El modelo de la traducción. IV Ética Discursiva y Diálogo Intercultural. Ética Intercultural Ensayos de una ética discursiva para contextos culturales conflictivos. (Re) Lecturas del pensamiento latinoamericano (pp 152 - 155). Quito, Ecuador, Ediciones Abya-Yala.

Sandoval, S., Madres Cuidadoras de la Cultura Qom, Inda, C., y Portal, G. (2013). Memoria y territorio vivido. Las prácticas de maternaje de las Madres Cuidadoras de la Cultura Qom. En: Experiencias en Educación Bilingüe Intercultural con las Madres Cuidadoras de la Cultura Qom. (pp.50-59), Resistencia: UNNE- Contexto.

Sandoval, S. (2018). Reconstrucción de la memoria histórica de la Comunidad Qom de Pampa del Indio. Enfoque intercultural. Universidad Nacional del Nordeste. Argentina. Tesis doctoral. Recuperado de https://repositorio.unne.edu.ar/xmlui/handle/123456789/9041

Silva, M. (2005). Mensajes del Gran Chaco, Literatura oral indígena. Resistencia, Chaco: Librería de la Paz. 